\title{
Effects of Verapamil on Ventricular Tachycardias Possibly Caused by Reentry, Automaticity, and Triggered Activity
}

\author{
Ruey J. Sung, William A. Shapiro, Edward N. Shen, Fred Morady, \\ and Jesse DAvis, Clinical Cardiac Electrophysiology Laboratory, \\ Cardiovascular Research Institute, and the Division of Cardiology, \\ Department of Medicine, University of California, \\ San Francisco, California 94143
}

A B S T R A C T To define the role of verapamil in the treatment of ventricular tachycardia (VT), we studied 21 patients with chronic recurrent VT. Electrophysiologic studies were performed before and during intravenous infusion of verapamil $(0.15 \mathrm{mg} / \mathrm{kg}$ followed by $0.005 \mathrm{mg} / \mathrm{kg}$ per $\mathrm{min}$ ). On the basis of the mode of VT initiation and termination, we identified three groups of patients: (a) 11 patients had VT suggestive of reentry, as VT could be initiated with ventricular extrastimulation and terminated with overdrive ventricular pacing. Verapamil did not affect the inducibility and cycle length of VT. (b) 7 patients had VT suggestive of catecholamine-sensitive automaticity as VT could not be initiated with programmed electrical stimulation but could be provoked by isoproterenol infusion. Moreover, the VT could not be converted to a sustained sinus rhythm with overdrive ventricular pacing and it resolved only with discontinuing isoproterenol infusion. Verapamil exerted no effects on VT. (c) 3 patients had VT with electrophysiologic characteristics suggestive of triggered activity related to delayed afterdepolarizations. Characteristically, after attaining a range of cycle lengths, the sinus, atrial or ventricular paced rhythm could initiate VT without ventricular extrastimulation. The first beat of VT invariably occurred late in the cardiac cycle with a premature coupling interval $0-80 \mathrm{~ms}$ shorter than the preceding QRS cycle length; the premature coupling interval gradually decreased as the sinus, atrial or ventricular paced cycle length progressively shortened. Of

This work was presented in part at the National Meeting of American Federation for Clinical Research in Washington, DC, May 1982.

Dr. Shen had a research fellowship sponsored by the Medical Research Council of Canada. Address correspondence to Dr. Sung.

Received for publication 8 July 1982 and in revised form 18 March 1983. note, verapamil completely suppressed VT inducibility in these three patients. These observations lead us to suggest that verapamil does not affect VT caused by reentry and catecholamine-sensitive automaticity but is effective in suppressing VT caused by triggered activity related to delayed afterdepolarizations in humans.

\section{INTRODUCTION}

Although clinical and electrophysiologic studies (1-6) have demonstrated that verapamil is an effective antiarrhythmic agent for supraventricular tachyarrhythmias, its role in the treatment of ventricular arrhythmias has not been defined. Wellens et al. (7) noted that verapamil exerted no antiarrhythmic effect in four patients with chronic recurrent ventricular tachycardia, whereas other investigators $(1,8-12)$ have reported that verapamil reduced ventricular ectopy and even abolished recurrent or life threatening ventricular tachyarrhythmias. These contradictory observations prompted us to assess the efficacy of verapamil for control of ventricular tachycardia.

We studied 21 patients whose ventricular tachycardia could be reproduced in our cardiac electrophysiology laboratory. Effects of verapamil on ventricular tachycardia were analyzed according to the mode of arrhythmia initiation and termination. Our findings suggest that among the various mechanisms of cardiac arrhythmias: reentry, automaticity, and triggered activity (13-15), verapamil is only effective in suppressing ventricular tachycardia with electrophysiologic characteristics consistent with triggered activity related to delayed afterdepolarizations.

\section{METHODS}

Study patients. 39 patients with clinical evidence of recurrent sustained ventricular tachycardia (lasting longer 
TABLE I

Clinical Profile

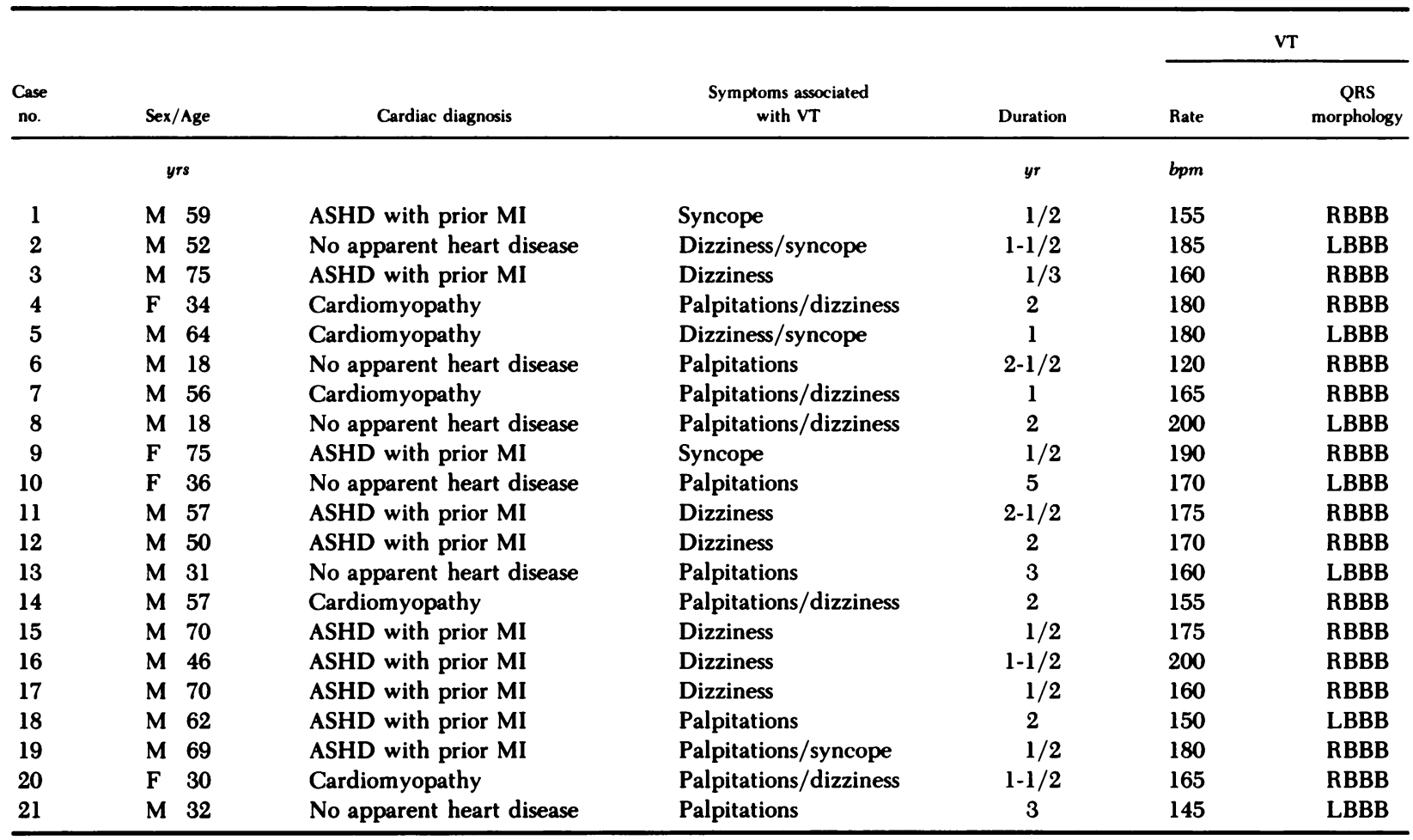

Abbreviations: ASHD, arteriosclerotic heart disease; bpm, beats per minute; LBBB, left bundle branch block pattern; MI, myocardial infarction; RBBB, right bundle branch block pattern; VT, ventricular tachycardia.

than $30 \mathrm{~s}$ or requiring electrical or pharmacologic intervention because of circulatory collapse) were evaluated in our electrophysiology laboratory. We excluded 18 patients in whom direct current countershock was required to terminate the arrhythmia during the control study. The remaining 21 patients constituted the study population. These 21 patients had reproducible ventricular tachycardia that lasted longer than $30 \mathrm{~s}$ or could be terminated by overdrive ventricular pacing. There were 17 men and 4 women ranging in age from 18 to $75 \mathrm{yr}$ (mean of 50.5). Their clinical data are summarized in Table I. Nine patients had arteriosclerotic heart disease with myocardial infarction of $>6$ mo before the study, five patients had cardiomyopathy, and the remaining seven patients had no clinical evidence of organic heart disease except for the rhythm disturbance.

Electrophysiology study. All patients gave informed consent, and all antiarrhythmic drugs were discontinued for at least over five half-lives before the study. The study was performed with patients in a postabsorptive, nonsedated state. With the conventional method (16), a quadripolar electrode catheter was introduced from the right femoral vein and placed in the right atrium for recording the His bundle potential (HBE). ${ }^{1}$ Another quadripolar electrode catheter was introduced through the right femoral vein and placed in the high right atrium (HRA) or right ventricle for

\footnotetext{
${ }^{1}$ Abbreviations used in this paper: $\mathrm{HBE}$, His bundle potential; HRA, high right atrium.
}

programmed electrical stimulation. Intracardiac electrograms were simultaneously displayed with electrocardiogram leads $V_{1}, I$, and III on a multichannel oscilloscope (Electronics for Medicine, VR-12, White Plains, NY), and were recorded at a paper speed of $50-100 \mathrm{~mm} / \mathrm{s}$ using filter frequency settings of $30-500 \mathrm{~Hz}$.

Protocol for programmed electrical stimulation. A programmed digital stimulator (Bloom \& Associates, Narberth, PA) was used to deliver electrical impulses of 2.0-ms duration of approximately twice diastolic threshold.

Incremental pacing. The high right atrium or right ventricle was paced for 5-10 beats with abrupt cessation. We began pacing with a cycle length slightly shorter than that of the sinus rhythm. After each burst of pacing we decreased the pacing cycle length by $10-20 \mathrm{~ms}$. The shortest pacing cycle length was $250 \mathrm{~ms}$.

Extrastimulation. Following incremental pacing, the high right atrium or right ventricle was driven at one or more cycle lengths $\left(S_{1}-S_{1}\right)$ (usually 600 and $400 \mathrm{~ms}$ ), and an atrial or ventricular premature beat $\left(S_{2}\right)$ was introduced from late diastole at progressively shorter coupling intervals $\left(S_{1}-S_{2}\right)$ after every eighth basic drive beat until atrial or ventricular refractoriness was encountered. During ventricular extrastimulation, if a single ventricular premature beat $\left(S_{2}\right)$ failed to elicit ventricular tachycardia, timed double and triple ventricular premature beats were delivered. The protocol was continued until it evoked a ventricular tachycardia or until all extrastimuli failed to evoke ventricular responses.

Programmed electrical stimulation as described above was 
performed in the left ventricle whenever it was necessary to induce arrhythmia.

After initiation of ventricular tachycardia, we delivered timed ventricular premature stimuli and/or short bursts of rapid (overdrive) ventricular pacing to terminate the arrhythmia.

Provocation of ventricular tachycardia with isoproterenol. If programmed electrical stimulation failed to induce ventricular tachycardia, isoproterenol was infused intravenously at $1-4 \mu \mathrm{g} / \mathrm{min}$ until the sinus rate was accelerated to a maximum of $150 \mathrm{beats} / \mathrm{min}$. If isoproterenol did not provoke an onset of ventricular tachycardia, programmed electrical stimulation as described above was then repeated during isoproterenol infusion (17).

Intravenous administration of verapamil. Following termination of ventricular tachycardia, intravenous verapamil was administered during sinus rhythm $(0.15 \mathrm{mg} / \mathrm{kg}$ infused over $2 \mathrm{~min}$ as the loading dose followed by continuous infusion at $0.005 \mathrm{mg} / \mathrm{kg}$ per $\mathrm{min}$ ). During verapamil infusion, programmed electrical stimulation protocol was repeated or intravenous infusion of isoproterenol was reinstituted to assess effects of verapamil. Serial blood samples were drawn for measurements of plasma verapamil concentration (18) immediately, $5 \mathrm{~min}, 15 \mathrm{~min}$, and $30 \mathrm{~min}$ after the loading injection. The plasma verapamil concentrations peaked at the end of the loading phase ranging from 132 to 286 (mean, $161 \pm 35) \mathrm{ng} / \mathrm{ml}$, then leveled off during the continuous infusion phase with a mean concentration of $126 \pm 22 \mathrm{ng} / \mathrm{ml}$.

Classification of mechanisms of ventricular tachycardia. Mechanisms of ventricular tachycardia can only be presumptive during electrophysiologic studies in humans (19, 20). The following electrophysiologic principles (13-15) were applied.

Reentry requires the presence of a conduction circuit with two pathways differing in electrophysiologic properties. A ventricular premature impulse may thus induce conduction block in one pathway and traverse slowly in the other pathway. If conduction in this latter pathway is sufficiently slow, the previously blocked pathway may recover excitability, allowing the impulse to traverse in a retrograde direction and initiating a reentrant phenomenon. When this phenomenon is perpetuated, a circus movement (tachycardia) is established. Therefore, ventricular tachycardia of a reentrant mechanism is suggested by the ability to reproducibly initiate the tachycardia with ventricular extrastimulation and/or rapid ventricular pacing. Furthermore, a reentrant ventricular tachycardia may be interrupted by timed extrastimulation and/or overdrive ventricular pacing, assuming the electrical impulses penetrate and alter the refractory periods of the tissues involved.

Automaticity is caused by spontaneous impulse initiation that results from gradual diastolic phase 4 depolarization. This may occur in both normal and partially depolarized fibers. Therefore, ventricular tachycardia of an automatic mechanism occurs spontaneously. Programmed electrical stimulation is not expected to initiate this arrhythmia, and timed ventricular extrastimulation or overdrive ventricular pacing is not expected to convert this arrhythmia to a sustained sinus rhythm $(13-15,20)$. Because of limitations of the study technique, no attempt was made to distinguish normal from abnormal automaticity.

Triggered activity related to delayed afterdepolarizations does not occur spontaneously and is dependent upon previous depolarizations for its initiation. A delayed afterdepolarization occurs after phase 3 repolarization has restored maximum diastolic potential. The delayed afterdepolarization may reach threshold and initiate an action potential during a critical range of cycle lengths. Generation of a train of such triggered action potentials can thus lead to an arrhythmia. Ventricular tachycardia caused by this mechanism may have some of the following electrophysiologic characteristics as suggested by Rosen and $\operatorname{Reder}(15):(a)$ the first beat of tachycardia usually appears late in the cardiac cycle; $(b)$ its initiation is related to a critical range of QRS cycle lengths (usually short rather than long cycle lengths); (c) its onset is more easily triggered by multiple rather than single ventricular depolarizations; and $(d)$ the interval between the first beat of tachycardia and its preceding sinus or paced beat (QRS complex) gradually decreases as the driving cycle length (sinus or paced beats) progressively shortens.

\section{RESULTS}

All 21 patients had reproducible ventricular tachycardia identical to that observed during spontaneous episodes. 14 of them had ventricular tachycardia of the right bundle branch block pattern and seven had ventricular tachycardia of the left bundle branch block pattern. The rates of ventricular tachycardia ranged from 120 to 200 beats/min. Based on the mode of arrhythmia initiation and termination, effects of verapamil on ventricular tachycardia were analyzed. We identified the following three groups of patients.

Ventricular tachycardia presumed to be due to reentry. 11 patients (cases $1,3-5,9,12,14-17$, and 19) had ventricular tachycardia with electrophysiologic characteristics suggestive of reentry. Of these patients, ventricular tachycardia could not be initiated from the atrium but could be initiated with programmed right ventricular extrastimulation. Five patients required single ventricular extrastimuli $\left(S_{2}\right)$; four patients, two ventricular extrastimuli; and two patients, three ventricular extrastimuli to provoke the tachycardia. The tachycardia zone could be determined in four of the five patients who required only single ventricular extrastimuli $\left(\mathrm{S}_{2}\right)$ to initiate the tachycardia (Fig. 1, $A$ and $B$ ). In these four patients, the tachycardia could be readily terminated by overdrive ventricular pacing (Fig. 2). Of note, there was progressive lengthening of the interval between the initiating ventricular premature beat $\left(S_{2}\right)$ and the first beat of tachycardia in response to increasing prematurity of ventricular extrastimulation (Fig. $1, A$ and $B)$. In the remaining seven patients, the tachycardia zone was not determined, as they required many trials of overdrive ventricular pacing to terminate the tachycardia.

With intravenous infusion of verapamil, the ventricular tachycardia remained inducible with programmed right ventricular extrastimulation, and the rates of ventricular tachycardia remained unchanged in all 11 patients. Furthermore, verapamil did not affect the tachycardia zone in the four patients in whom the zone could be determined. Three patients (cases 5,9 , and 19) required direct current countershock to 

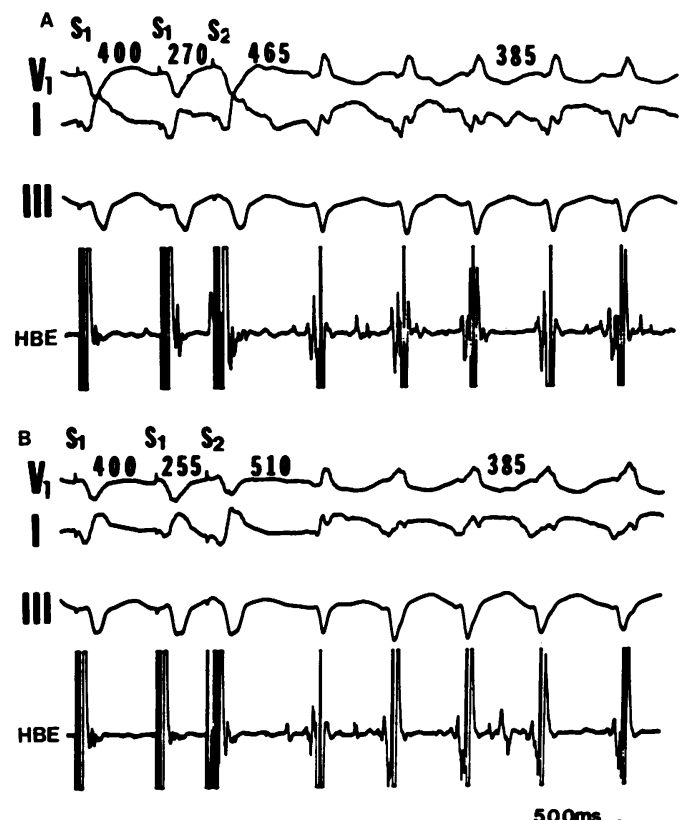

Figure l Initiation of ventricular tachycardia with programmed ventricular extrastimulation in a 59-yr-old man with arteriosclerotic heart disease. During right ventricular pacing at a cycle length $\left(S_{1}-S_{1}\right)$ of $400 \mathrm{~ms}$, a ventricular premature beat $\left(S_{2}\right)$ with a premature coupling interval $\left(S_{1}-\right.$ $\mathrm{S}_{2}$ ) of $270 \mathrm{~ms}$ initiates an onset of ventricular tachycardia with a cycle length of $385 \mathrm{~ms}(A)$. Further shortening of the ventricular premature coupling interval $\left(S_{1}-S_{2}\right)$ to $255 \mathrm{~ms}$ can also initiate the onset of ventricular tachycardia $(B)$. Note that the shorter the ventricular premature coupling interval $\left(S_{1}-S_{2}\right)$, the longer the interval between the ventricular premature beat $\left(S_{2}\right)$ and the first beat of ventricular tachycardia (465 vs. $510 \mathrm{~ms}$ ). Electrocardiogram leads $V_{1}$, I, and III are simultaneously recorded with HBE in this and subsequent figures.

terminate the ventricular tachycardia because of verapamil-induced hypotension.

Ventricular tachycardia presumed to be due to automaticity. In seven patients (cases $2,7,8,11,13,18$,
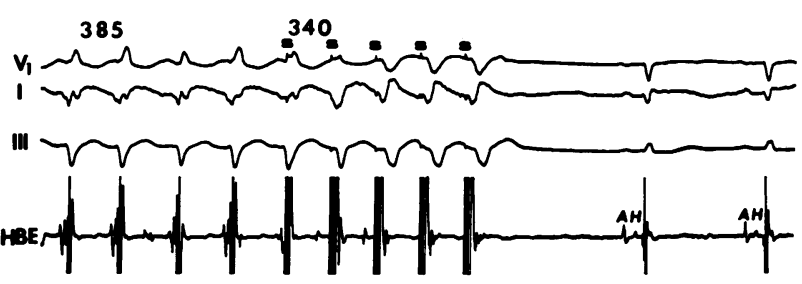

S000m

Figure 2 Termination of sustained ventricular tachycardia with overdrive ventricular pacing (same patient as in Fig. 1). Right ventricular pacing at a cycle length (S-S) of 340 ms for five beats converts the ventricular tachycardia with a cycle length of $385 \mathrm{~ms}$ to sinus rhythm. A and $\mathrm{H}$ are atrial and $\mathrm{His}$ bundle electrograms, respectively. and 20) in whom ventricular tachycardia could not be elicited by programmed electrical stimulation from the right atrium and both ventricles, isoproterenol infusion at $2-4 \mu \mathrm{g} / \mathrm{min}$ could by itself provoke an onset of ventricular tachycardia (Fig. 3). In contrast to reentrant ventricular tachycardia, ventricular tachycardia so induced could not be converted to a sustained sinus rhythm with overdrive ventricular pacing. One example is illustrated in Fig. 4. In each instance, ventricular tachycardia resolved after discontinuation of isoproterenol infusion.

During intravenous infusion of verapamil, institution of isoproterenol infusion at the same dosage of $2-4 \mu \mathrm{g} / \mathrm{min}$ invariably provoked the same form of ventricular tachycardia at the same rate in all seven patients. Verapamil thus appeared to exert no antiarrhythmic effects in this group of patients.

Ventricular tachycardia with electrophysiologic characteristics consistent with triggered activity related to delayed afterdepolarizations. In the remaining three patients (cases 6,10 , and 21), the mode of initiation of ventricular tachycardia was distinctly different from those described above. In each patient the tachycardia could be initiated by accelerating the sinus rate, however, if the sinus rate was accelerated beyond a critical range, the tachycardia could no longer be initiated (Fig. 5, A-D). This phenomenon could be reproducibly demonstrated either with hyperventilation or with handgrip. The critical range of sinus cycle lengths that initiated ventricular tachycardia varied slightly each day and it measured 520-720, 490-530, and 450-710 ms, respectively, for the three patients (cases 6, 10, and 21). Furthermore, the first beat of ventricular tachycardia usually occurred late in the cardiac cycle: $\mathbf{0 - 8 0} \mathrm{ms}$ shorter than the preceding sinus cycle length. During verapamil infusion, repeated hyperventilation and handgrip could no longer initiate the arrhythmia despite acceleration of the sinus rate with cycle lengths to be within the critical range in all three patients (Fig. 5, E and F). None of these three patients were taking digitalis, and these findings were not observed in either group I or group II patients.

During electrophysiologic studies, atrial or ventricular extrastimulation did not elicit ventricular tachycardia. However, incremental atrial or ventricular pacing for 5-10 beats within the similar range of cycle lengths reproducibly initiated ventricular tachycardia (Figs. 6 and 7). The interval between the first beat of ventricular tachycardia and the last paced beat (QRS complex) was also noted to gradually decrease as the paced cycle length ( $R-R$ interval) progressively shortened (Figs. 6, 7, and 8). Of note, overdrive atrial or ventricular pacing with a ventricular cycle length shorter than the critical range could terminate the 

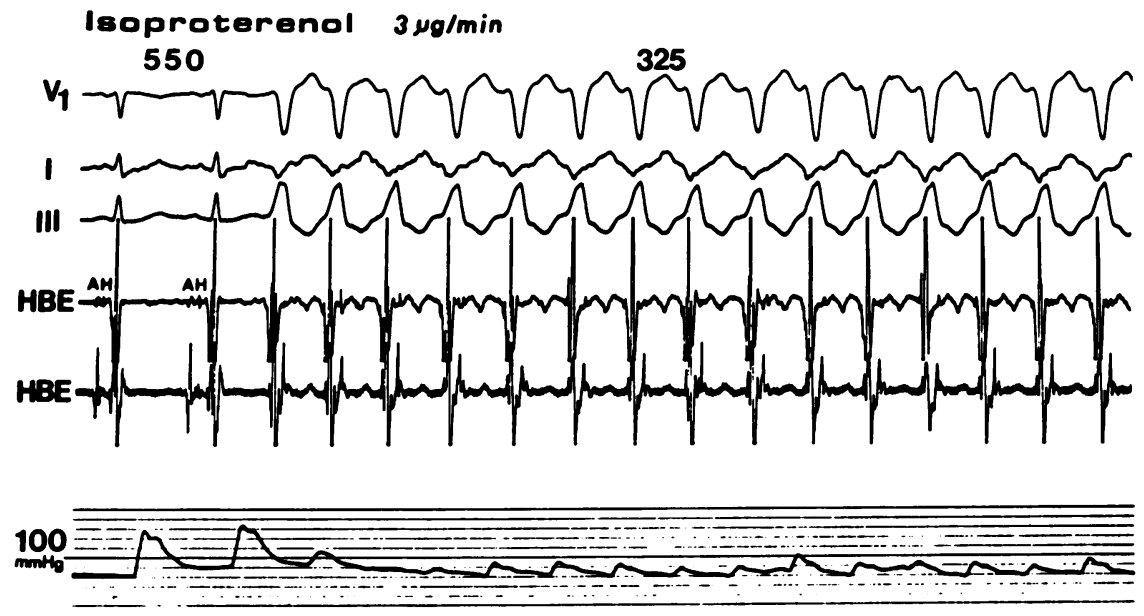

FIGURE 3 Induction of a paroxysm of ventricular tachycardia with intravenous infusion of isoproterenol in a 52-yr-old man with no clinical evidence of organic heart disease. Following failure of programmed electrical stimulation to induce arrhythmia, isoproterenol infusion at a dosage of $3 \mu \mathrm{g} / \mathrm{min}$ accelerates the sinus rate from 75 to 109 beats/min (cycle length 550 $\mathrm{ms}$ ) and provokes an onset of ventricular tachycardia with a cycle length of $325 \mathrm{~ms}$. Right femoral arterial pressure is recorded at the bottom.

ventricular tachycardia. By contrast, incremental atrial or ventricular pacing did not induce the arrhythmia in either group I or group II patients.

During intravenous verapamil infusion, early development of atrioventricular nodal block precluded attainment of the critical range of cycle lengths $(R-R$ interval) with atrial pacing. Thus, the effects of verapamil on the initiation of ventricular tachycardia during atrial pacing could not be assessed. Nevertheless, ventricular pacing within the critical range of cycle lengths could no longer initiate the arrhythmia in all three patients (Fig. 9).

\section{DISCUSSION}

The results of this study indicate that intravenous verapamil at concentrations of $126 \pm 22 \mathrm{ng} / \mathrm{ml}$ does not exert antiarrhythmic effects on ventricular tachycardia with a mode of initiation and termination suggestive of either reentry or catecholamine-sensitive automaticity as the underlying mechanism. However, verapamil at these concentrations is effective in suppressing ventricular tachycardia with electrophysiologic characteristics consistent with triggered activity related to delayed afterdepolarizations $(13-15,20)$.
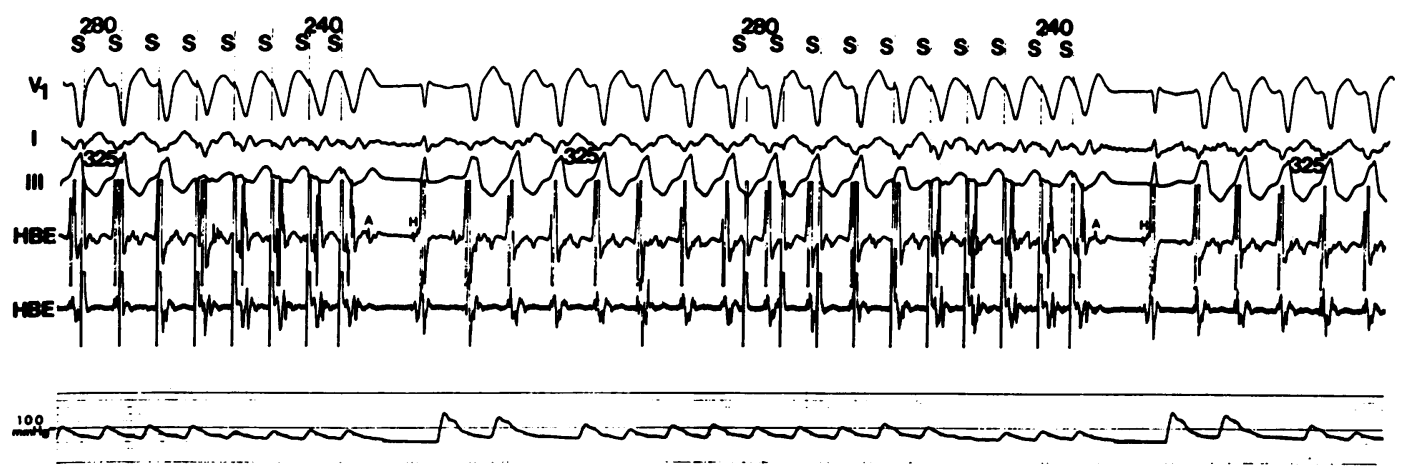

FIGURE 4 Response of isoproterenol-induced ventricular tachycardia to overdrive ventricular pacing (same patient as in Fig. 3). The ventricular tachycardia has a cycle length of $325 \mathrm{~ms}$ as shown in Fig. 3. Right ventricular overdrive pacing with a cycle length of $280 \mathrm{~ms}$ for 7 to 9 beats followed by a ventricular premature beat with a premature coupling interval of 240 ms can only temporarily suppress the ventricular tachycardia as the arrhythmia emerges immediately after each sinus beat. Right femoral arterial pressure is recorded at the bottom. 

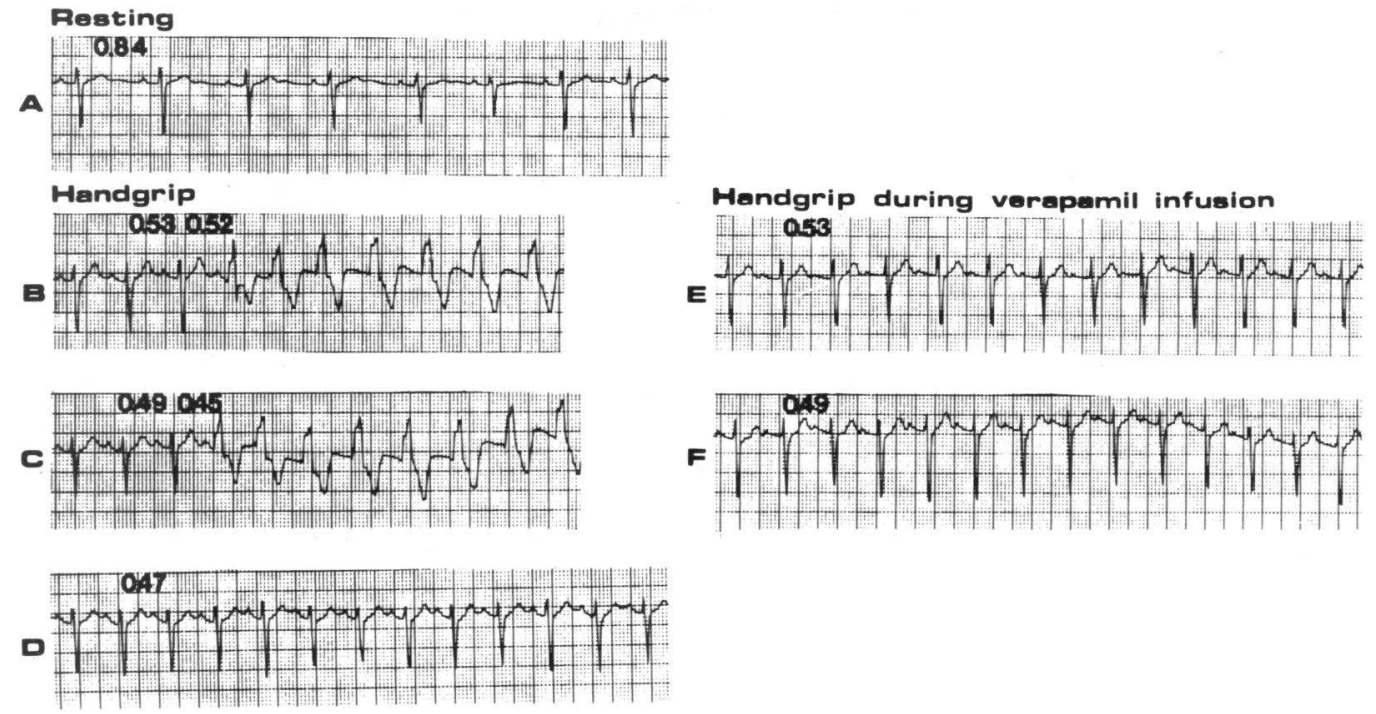

FIGURE 5 Cycle length dependency for the initiation of ventricular tachycardia during sinus rhythm in an 18-yr-old man with no clinical evidence of organic heart disease. At rest, the cycle length of sinus rhythm is $0.84 \mathrm{~s}(A)$. The sinus rate then accelerates during handgrip. Ventricular tachycardia is initiated when the sinus rhythm attains cycle lengths between 0.53 and $0.49 \mathrm{~s}(B$ and $C)$. Further acceleration of the sinus rate (cycle length of $0.48 \mathrm{~s}$ or shorter) initiates no ventricular tachycardia $(D)$. Note that the interval between the first beat of ventricular tachycardia and its preceding sinus beat (QRS complex) shortens $(0.52 \rightarrow 0.45 \mathrm{~s})$ as the sinus rate accelerates $(B$ and $C)$. During verapamil infusion, repeated handgrip accelerates the sinus rate with cycle lengths between 0.53 and $0.49 \mathrm{~s}$ but fails to initiate ventricular tachycardia $(E$ and $F)$.

Ventricular tachycardia due to triggered activity related to delayed afterdepolarizations. Triggered activity related to delayed afterdepolarizations has been identified and characterized in both isolated animal and human tissues (21-29). It has been observed in the presence as well as the absence of digitalis intoxication. However, the mechanism by which delayed afterdepolarizations occur is still incompletely understood. Experimental studies have led other investigators to suggest that these afterdepolarizations occur after a transient current carried by sodium and modulated by calcium (30-32). By inhibiting the calciumdependent slow channel response (33-35), verapamil may suppress these delayed afterdepolarizations (2529). In keeping with these findings, we suggest that verapamil may prevent initiation of ventricular tachycardia with electrophysiologic characteristics consistent with triggered activity related to delayed afterdepolarizations in man. Of note was the observation that all three patients who had ventricular tachycardia suggestive of triggered activity had no clinical evidence of organic heart disease.

Ventricular tachycardia suggestive of triggered activity (group III patients) conforms to some of the elec- trophysiologic features described in isolated tissue preparations $(15,21-29)$. However, we realize that differentiation between reentry and triggered activity remains difficult. This is because both reentry and triggered activity may have similar clinical electrophysiologic manifestations despite having differences in their basic electrophysiologic mechanisms.

(a) Delayed afterdepolarizations do not occur spontaneously. Their occurrence depends on previous depolarizations (15). Similar to reentry, triggered activity can, therefore, be initiated by programmed electrical stimulation. It may be initiated by both atrial and ventricular pacing as demonstrated in the present study. In contrast, a reentrant ventricular tachycardia is rarely initiated from the atrium $(36,37)$.

(b) The amplitude of delayed afterdepolarizations increases as the basic driving cycle length shortens (15). The initiation of an onset of triggered activity is thus a cycle length-dependent phenomenon. Nevertheless, shortening of the basic driving cycle length may induce unidirectional block in one pathway and slow conduction in the other pathway, setting up conditions for reentry to occur $(13,15,20)$.

(c) Overdrive pacing may penetrate and alter re- 

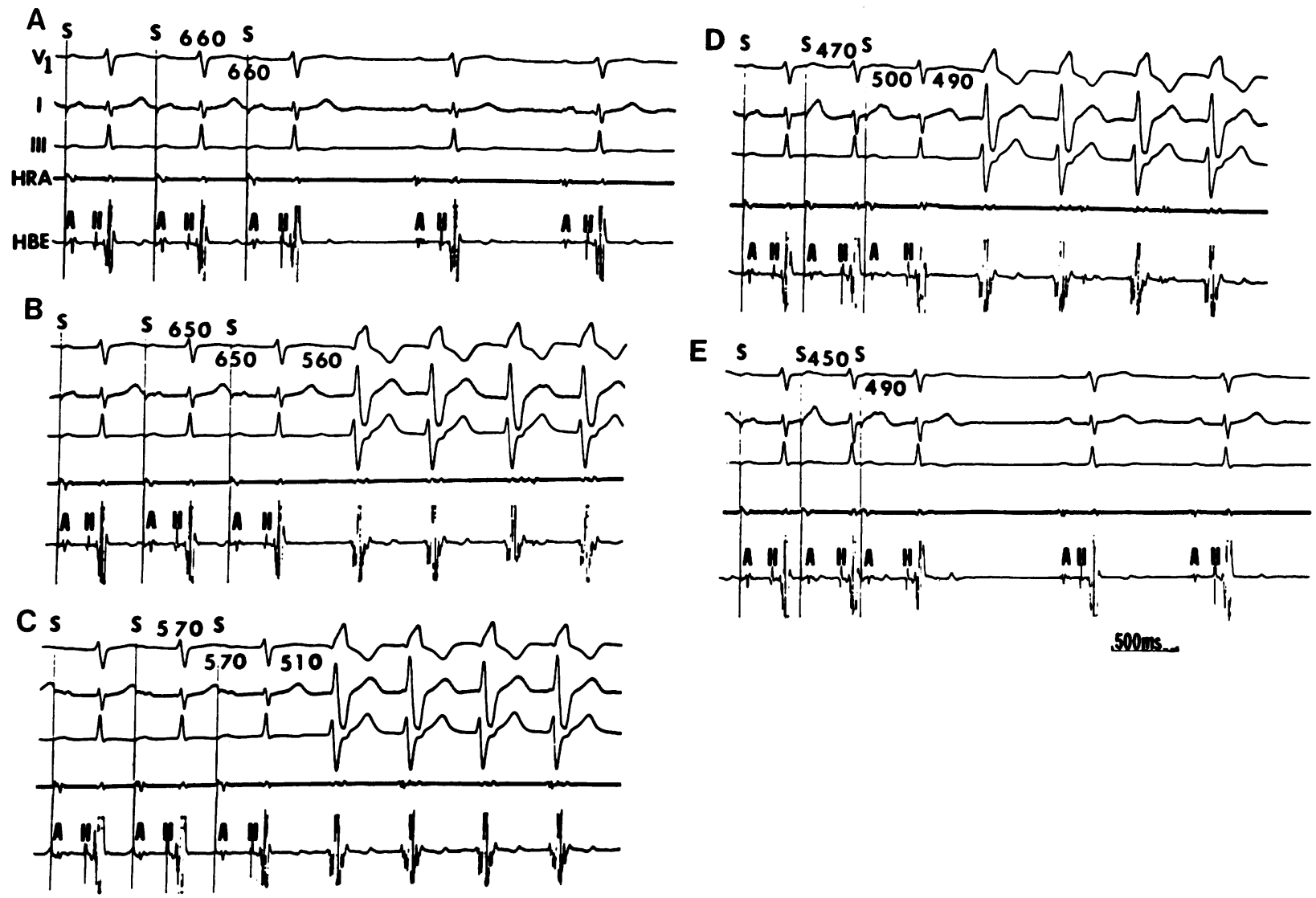

FIGURE 6 Induction of ventricular tachycardia during atrial paced rhythms (same patient as in Fig. 5). (A) HRA pacing at a cycle length (S-S) of 660 ms (corresponding $R-R$ interval, 660 ms) initiates no ventricular tachycardia. $(B, C, D)$ HRA pacing at cycle lengths (S-S) of 650 , 570 , and $470 \mathrm{~ms}$, respectively (corresponding $R-R$ intervals of 650,570 , and $500 \mathrm{~ms}$, respectively) initiates an onset of ventricular tachycardia. $(E)$ HRA pacing at a cycle length (S-S) of $450 \mathrm{~ms}$ (corresponding $\mathrm{R}-\mathrm{R}$ interval, $490 \mathrm{~ms}$ ) initiates no ventricular tachycardia. Note that the interval between the first beat of ventricular tachycardia and its preceding atrial paced beat (QRS complex) gradually shortens as the atrial pacing rate progressively increases $(B, C$, and $D)$.

fractoriness of reentrant pathways, thereby terminating a reentrant arrhythmia. On the other hand, overdrive pacing can cause enhancement of electrogenic sodium extrusion, which hyperpolarizes the membrane potentials and may thereby abolish triggered activity (38).

(d) As the basic driving cycle length shortens, the interval between the last paced (initiating) beat and the first beat of arrhythmia tends to increase in reentry, whereas it tends to decrease in triggered activity. Exceptions to the rule, however, may occur. In reentry, this interval presumably reflects total conduction time in both the antegrade and retrograde limbs of the reentrant circuit. If the retrograde limb conducts rapidly because of early recovery of excitability at shorter cycle lengths, the total conduction time may remain unchanged or even decrease rather than increase. Furthermore, in triggered activity, the first delayed afterdepolarization may paradoxically diminish its amplitude and become subthreshold as the basic driving cycle length shortens. The subsequent emergence of the second delayed afterdepolarization may then manifest with an unexpected prolongation of the premature coupling interval (15). Therefore, the interval between the initiating beat and the first beat of arrhythmia alone may not be a strong indicator for either reentry or triggered activity.

Ventricular tachycardia due to reentry. It has been 

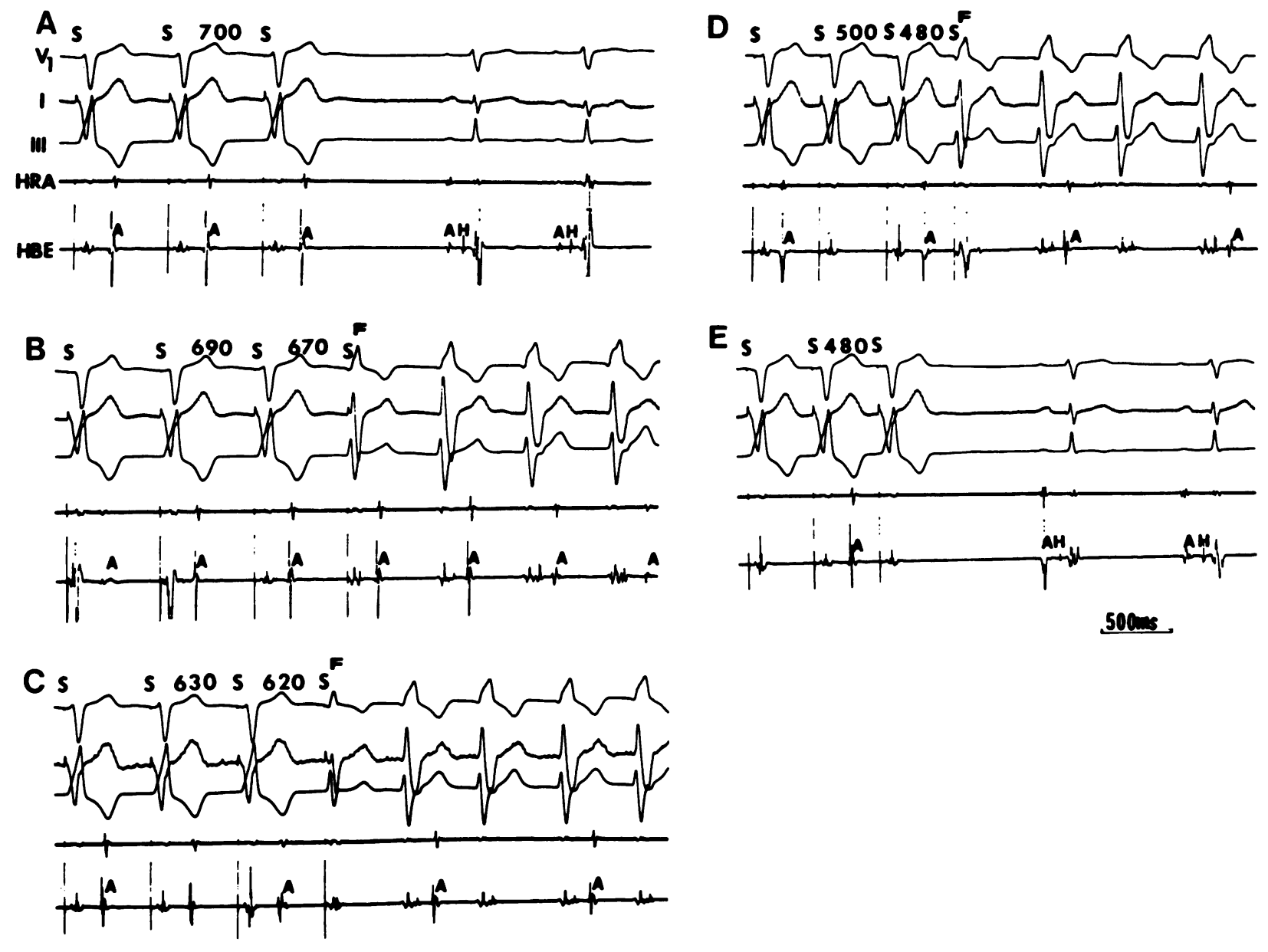

FIGURE 7 Induction of ventricular tachycardia during ventricular paced rhythm (same patient as in Fig. 5). (A) Right ventricular pacing at a cycle length (S-S) of $700 \mathrm{~ms}$ initiates no ventricular tachycardia. $(B, C, D)$ Right ventricular pacing at cycle lengths of 690,630 , and $500 \mathrm{~ms}$, respectively, initiates an onset of ventricular tachycardia. $(E)$ Right ventricular pacing at a cycle length of $480 \mathrm{~ms}$ initiates no ventricular tachycardia. Note that the interval between the first beat of ventricular tachycardia and its preceding ventricular paced beat gradually shortens as the ventricular pacing rate progressively increases $(B, C$, and $D) . F$, ventricular fusion.

suggested that the slow channel action potentials may become dominant under pathologic conditions, such as myocardial ischemia, in regions of the heart ordinarily dominated by the fast sodium channel response $(39,40)$. Such slow channel action potentials have been implicated in the genesis of reentrant ventricular arrhythmias because of their propensity for very slow propagation of impulses $(41,42)$. In chronic infarcted canine hearts with inducible reentrant ventricular arrhythmia, El-Sherif and Lazzara (43) noted that verapamil could suppress the arrhythmia, whereas Davis et al. (44) found that verapamil was totally ineffective. However, El-Sherif and Lazzara (43) postulated that the depressed part with slow conduction in the reentrant circuit was caused by the depressed fast sodium channel rather than the calcium-dependent slow channel response, and that verapamil probably exerted its antiarrhythmic effect by improving conduction in the depressed cells that formed part of the reentrant circuit.

In keeping with the findings of Wellens et al. (7) this study demonstrated that verapamil was ineffective in suppressing reentrant ventricular tachycardia in man. Because of this finding, it is tempting to speculate that the part with slow conduction in the diseased ventricular myocardium probably results from a depressed 


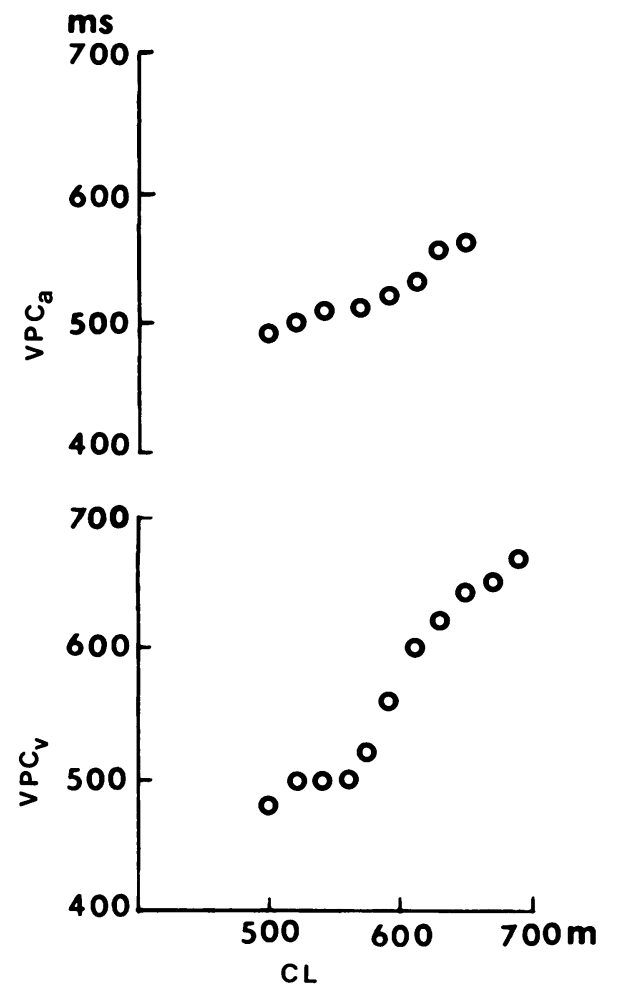

Figure 8 Prematurity of the first beat of ventricular tachycardia induced during atrial and ventricular paced rhythms (same patient as in Fig. 5). The interval between the first beat of ventricular tachycardia and its preceding atrial paced beat (QRS complex) is designated as $V P C_{a}$ (Fig. 6) and that between the first beat of ventricular tachycardia and its preceding ventricular paced beat (Fig. 7) is designated as $V P C_{v}$. Both $V P C_{a}$ and $V P C_{v}$ are plotted against the driving cycle length $\left(C L ; \mathrm{R}-\mathrm{R}\right.$ interval). Note that the $V P C_{a}$ and $V P C_{v}$ intervals gradually shorten as the driving cycle length $(C L)$ progressively decreases.

fast sodium channel rather than the calcium-dependent slow channel response $(45,46)$ in our group I patients.

Ventricular tachycardia due to catecholamine-sensitive automaticity. It has also been postulated that the calcium-dependent slow channel response may become dominant, and, in turn, may give rise to an automatic rhythm under pathologic conditions (39, 40). In this situation, verapamil would be expected to be effective in suppressing such an automatic rhythm. The negative finding of our study suggests that automatic ventricular tachycardia provocable with isoproterenol is probably not of a form of automatic rhythm caused by the calcium-dependent slow channel response. Unfortunately, because of the limitations of the study technique, we were not able to make dif-
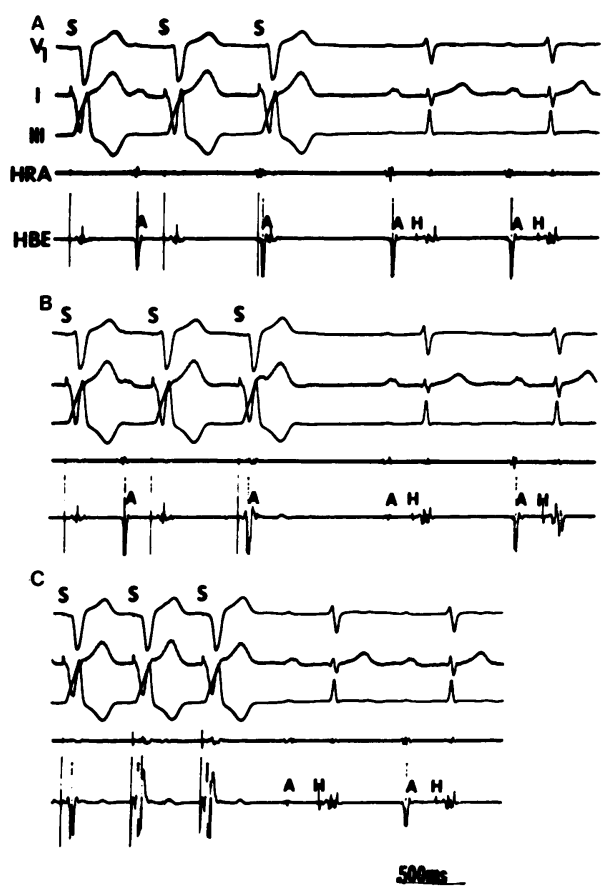

FIgURE 9 Suppression of induction of ventricular tachycardia during ventricular paced rhythm with verapamil infusion (same patient as in Fig. 5). During intravenous infusion of verapamil $(0.15 \mathrm{mg} / \mathrm{kg}$ followed by $0.005 \mathrm{mg} / \mathrm{kg}$ per $\mathrm{min})$, right ventricular pacing at cycle lengths $(\mathrm{S}-\mathrm{S})$ of 690 $(A), 630(B)$, and $500(C) \mathrm{ms}$, respectively, initiates no ventricular tachycardia. Note that ventricular pacing at these cycle lengths initiates onset of ventricular tachycardia before verapamil infusion as shown in Fig. $7(B, C$, and $D)$.

ferentiation between normal and abnormal automaticity (13-15).

Clinical implications. Observations made in the present study have certain important implications regarding the diagnosis and treatment of ventricular tachycardia in humans: (a) Electrophysiologic characteristics of ventricular tachycardia vary among patients as observed during electrophysiologic studies. This suggests that they have different underlying mechanisms. (b) Differentiation between reentry and triggered activity remains difficult. While verapamil may be still useful for treating some forms of ventricular tachycardias caused by reentry and automaticity, it may be specifically effective in suppressing ventricular tachycardia caused by triggered activity related to delayed afterdepolarizations. Further studies are necessary to substantiate our findings. (c) Pharmacologic testing with various antiarrhythmic drugs such as fast sodium channel depressants (i.e., procainamide) and beta adrenergic blockers (i.e., propranolol) may 
help to further define the underlying mechanisms of ventricular tachycardia.

\section{ACKNOWLEDGMENT}

This work was supported in part by Harris $R$ Fund 25835, University of California, San Francisco, CA.

\section{REFERENCES}

1. Schamroth, L., D. M. Krikler, and C. Garrett. 1972. Immediate effects of intravenous verapamil in cardiac arrhythmias. Br. Med. J. 1:660-662.

2. Krikler, D. M., and R. A. J. Spurrell. 1974. Verapamil in the treatment of paroxysmal supraventricular tachycardia. Postgrad. Med. J. 50:447-453.

3. Wellens, H. J. J., S. L. Tan, F. W. H. Bar, D. R. Duren, K. I. Lie, and H. M. Dohmen. 1977. Effect of verapamil studied by programmed electrical stimulation of the heart in patients with paroxysmal re-entrant supraventricular tachycardia. Br. Heart J. 39:1058-1066.

4. Hagemeijer, F. 1978. Verapamil in the management of supraventricular tachyarrhythmias occurring after a recent myocardial infarction. Circulation. 57:751-755.

5. Sung, R. J., B. Elser, and R. G. McAllister Jr. 1980. Intravenous verapamil for termination of re-entrant supraventricular tachycardias. Intracardiac studies correlated with plasma verapamil concentrations. Ann. Intern. Med. 93:682-689.

6. Waxman, H. L., R. J. Myerburg, R. Appel, and R. J. Sung. 1981. Verapamil for control of ventricular rate in paroxysmal supraventricular tachycardia and atrial fibrillation or flutter. A double-blind randomized crossover study. Ann. Intern. Med. 94:1-6.

7. Wellens, H. J., F. W. Bar, K. I. Lie, D. R. Duren, and H. J. Dohmen. 1977. Effect of procainamide, propranolol and verapamil on mechanism of tachycardia in patients with chronic recurrent ventricular tachycardia. Am. J. Cardiol. 40:579-585.

8. Benter, F., and H. D. Reploh. 1968. Behandlung von Kammertachykardien mit isoptin. Med. Klin. 63:715717.

9. Gotsman, M. S., B. S. Lewis, A. Bakst, and A. S. Mitha. 1972. Verapamil in life-threatening tachyarrhythmias. S. Afr. Med. J. 46:2017-2019.

10. Fauchier, J. P., J. Lanfranchi, G. G. Ginies, and R. Raynaud. 1974. Syncope par "torsade de pointe" au cours d'un traitement par la chloroquine etude de l'electrogramme Hisien et traitement par le verapamil. Ann. Cardiol. Angeiol. 23:341-346.

11. Wu, D., H. C. Kuo, and J. S. Hung. 1981. Exercise-triggered paroxysmal ventricular tachycardia. A repetitive rhythmic activity possibly related to afterdepolarization. Ann. Intern. Med. 95:410-414.

12. Mason, J. W. 1982. Efficacy of verapamil in recurrent ventricular tachycardia. Am. J. Cardiol. 49:1015. (Abstr.)

13. Hoffman, B. F., and M. R. Rosen. 1981. Cellular mechanisms for cardiac arrhythmias. Circ. Res. 49:1-15.

14. Hoffman, B. F., and K. Dangman. 1982. Are arrhythmias caused by automatic impulse generation? In Normal and Abnormal Conduction In The Heart. A. Paes de Carvalho, B. F. Hoffman, and H. Lieberman, editors. Futura Publishing Co., Mount Kisco, New York. 24:429-460.

15. Rosen, M. R., and R. F. Reder. 1981. Does triggered activity have a role in the genesis of cardiac arrhythmias? Ann. Intern. Med. 94:794-801.

16. Scherlag, B. J., S. H. Lau, R. H. Helfant, W. D. Berkowitz, E. Stein, and A. N. Damato. 1969. Catheter technique for recording $\mathrm{His}$ bundle activity in man. Circulation. 39:13-19.

17. Reddy, C. P., and L. S. Gettes. 1979. Use of isoproterenol as an aid to electric induction of chronic recurrent ventricular tachycardia. Am. J. Cardiol. 44:705-713.

18. McAllister, R. G., Jr., and S. M. Howell. 1976. Fluorimetric assay of verapamil in biological fluids and tissues. J. Pharm. Sci. 65:431-432.

19. Wellens, H. J. J. 1978. Value and limitations of programmed electrical stimulation of the heart in the study and treatment of tachycardias. Circulation. 57:845-853.

20. Wellens, H. J. J., P. Brugada, E. J. D. M. Vanagt, D. L. Ross, and F. W. Bar. 1981. New studies with triggered automaticity. In Cardiac Arrhythmias. A Decade of Progress. D. C. Harrison, editor. G. K. Hall \& Co., Boston, MA. 44:601-610.

21. Rosen, M. R., H. Gelband, and B. F. Hoffman. 1973. Correlation between effects of ouabain on the canine electrocardiogram and transmembrane potentials of isolated Purkinje fibers. Circulation. 47:65-72.

22. Ferrier, G. R., J. H. Saunders, and C. Mendez. 1973. A cellular mechanism for the generation of ventricular arrhythmias by acetylstrophanthidin. Circ. Res. 32:600609.

23. Hashimoto, K., and G. K. Moe. 1973. Transient depolarizations induced by acetylstrophanthidin in specialized tissue of dog atrium and ventricle. Circ. Res. 32:618-624.

24. Ferrier, G. R. 1976. The effects of tension in acetylstrophanthidin-induced transient depolarizations and after contractions in canine myocardial and Purkinje tissues. Circ. Res. 38:156-162.

25. Wit, A. L., and P. F. Cranefield. 1976. Triggered activity in cardiac muscle fibers of the simian mitral valve. Circ. Res. 38:85-98.

26. Wit, A. L., and P. F. Cranefield. 1977. Triggered and automatic activity in the canine coronary sinus. Circ. Res. 41:435-445.

27. Hordof, A. J., A. Spotniz, L. Mary-Rabine, J. R. Malm, and M. R. Rosen. 1978. The cellular electrophysiologic effects of digitalis on human atrial fibers. Circulation. 57:223-229.

28. Wit, A. L., J. J. Fenoglio, Jr., A. J. Hordof, and K. Reemtsma. 1979. Ultrastructure and transmembrane potentials of cardiac muscle in the human anterior mitral valve leaflet. Circulation. 59:1284-1292.

29. Mary-Rabine, L., A. J. Hordof, P. Danilo Jr., J. R. Malm, and M. R. Rosen. 1980. Mechanisms for impulse initiation in isolated human atrial fibers. Circ. Res. 47:267277.

30. Aronson, R. S., and J. M. Gelles. 1977. The effect of ouabain, dinitrophenol and lithium on the pacemaker current in sheep cardiac Purkinje fibers. Circ. Res. 40:517-524.

31. Tsien, R., and D. O. Carpenter. 1978. Ionic mechanisms of pacemaker activity in cardiac Purkinje fibers. Fed. Proc. 37:2127-2131.

32. Kass, R., R. Tsien, and $R$. Weingart. 1978. Ionic basis of transient inward current induced by strophanthidin in cardiac Purkinje fibers. J. Physiol. (Lond.). 281:209226.

33. Tritthart, H., B. Fleckenstein, and A. Fleckenstein. 1971. 
Some fundamental actions of antiarrhythmic drugs on the excitability and the contractility of single myocardial fibers. Naunyn Schmiedebergs Arch. Pharmakol. 269:212-219.

34. Cranefield, P. F., R. S. Aronson, and A. L. Wit. 1974. Effect of verapamil on the normal action potential and on calcium-dependent slow response of canine cardiac Purkinje fibers. Circ. Res. 34:204-213.

35. Zipes, D. P., and J. C. Fischer. 1974. Effects of agents which inhibit the slow channel on sinus node automaticity and atrioventricular conduction in the dog. Circ. Res. 34:184-192.

36. Wellens, H. J. J., F. W. Bar, J. Farre, D. L. Ross, I. Wiener, and E. J. Vanagt. 1980. Initiation and termination of ventricular tachycardia by supraventricular stimuli. Incidence and electrophysiologic determinants as observed during programmed stimulation of the heart. Am. J. Cardiol. 46:576-582.

37. Zipes, D. P., P. R. Foster, P. J. Troup, and D. H. Pedersen. 1979. Atrial induction of ventricular tachycardia: reentry versus triggered automaticity. Am. J. Cardiol. 44:1-8.

38. Wit, A. L., P. F. Cranefield, and D. C. Gadsby. 1981 Electrogenic sodium extrusion can stop triggered activity in the canine coronary sinus. Circ. Res. 49:1029-1042.

39. Zipes, D. P., H. R. Besch Jr., and A. M. Watanabe. 1975. Role of the slow current in cardiac electrophysiology. Circulation. 51:761-765.
40. Rosen, M. R., A. L. Wit, and B. F. Hoffman. 1975. Electrophysiology and pharmacology of cardiac arrhythmias VI. Cardiac effects of verapamil. Am. Heart J. 89:665673.

41. Cranefield, P. F., A. L. Wit, and B. F. Hoffman. 1972. Conduction of the cardiac impulse. III. Characteristics of very slow conduction. J. Gen. Physiol. 59:227-232.

42. Wit, A. L., and J. T. Bigger Jr. 1975. Possible electrophysiological mechanisms for lethal arrhythmias accompanying myocardial ischemia and infarction. Circulation. 51(Suppl. III):III-96. (Abstr.)

43. El-Sherif, N., and R. Lazzara. 1979. Reentrant ventricular arrhythmias in the late myocardial infarction period. 7. Effect of verapamil and D-600 and the role of the "slow channel". Circulation. 60:605-615.

44. Davis, J., R. Glassman, and A. L. Wit. 1982. Method for evaluating the effects of antiarrhythmic drugs on ventricular tachycardia with different electrophysiologic characteristics and different mechanisms in the infarcted canine heart. Am. J. Cardiol. 49:1176-1184.

45. Spear, J. F., L. N. Horowitz, A. B. Hodess, H. MacVaugh III, and E. N. Moore. 1979. Cellular electrophysiology of human myocardial infarction. 1. Abnormalities of cellular activation. Circulation. 59:247-256.

46. Gilmour, R. F. Jr., E. N. Prystowsky, J. J. Heger, and D. P. Zipes. 1983. Cellular electrophysiologic abnormalities of diseased human ventricular myocardium. Am. J. Cardiol. 51:137-144. 